\title{
Barrier Coverage For Underwater Sensor Networks
}

\author{
Stanley Barr, Benyuan Liu, and Jie Wang \\ Computer Science Department \\ University of Massachusetts Lowell \\ Email: \{sbarr,bliu,wang $\} @$ cs.uml.edu
}

\begin{abstract}
Using sonar to detect submarines was an effective method. Current technologies, however, have made it possible for submarines to thwart standard (active or passive) sonar mechanisms [1]. Thus, finding alternatives to detect submarines becomes important and timely. One viable alternative is to use magnetic or acoustic sensors in close proximity of possible underwater pathways a submarine may pass through. This approach may require deploying large-scale underwater sensor networks to form strong barriers for coastline protection.

In this paper we first derive the notion of 3-dimensional stealth distance to measure how far a submarine can travel in a sensor network before being detected by a sensor. Next we prove that there is no strong barrier coverage in a large 3-dimensional fixed emplacement sensor field where sensor locations follow a Poisson point process, i.e., there always exists a path by which an adversary informed of the locations of the sensors can move without being detected. Finally, we describe an energy conserving approach to constructing a strong 3-dimensional barrier using mobile nodes so that intruding submarines cannot pass through without being detected.
\end{abstract}

\section{INTRODUCTION}

International submarine sales are not covered by any nonproliferation treaty on today's markets, making it possible for nation states with sufficient resources to purchase submarines, many of which are capable of launching cruise missiles to deliver conventional, nuclear, chemical, or biological payloads [2].

To make things worse, recent technology advances have also made it possible for submarines to evade standard sonar detection [1]. In particular, submarine hulls can be fitted with rubber anti-SONAR protection tiles to thwart active SONAR detection. These rubber tiles also dampen intra-submarine noise to thwart passive acoustic detection at a distance.

Thus, finding alternatives to detect submarines becomes important and timely. Using magnetic and/or acoustic enabled sensors in close proximity is a viable new approach. Recent advances in embedded systems, such as micro electro-mechanical systems and communications hardware, have made this approach more reliable and relatively inexpensive with lower power consumption [3]. Large-scale deployments of underwater wireless sensor networks are expected to become a reality in the near future.

This paper develops a framework capable of providing a strong barrier to detect intruding submarines in such a sensor network. Large-scale sensor deployments may occur by dispersal from an aircraft or artillery ordinance. Such deployment strategies can deploy a large number of sensors over a vast space quickly, but limit the control of sensor placement. In this paper we consider dispersal patterns whose result is a uniform distribution over a 3-dimensional (3D) space of interest. We start by developing the notion of 3D stealth distance. This is the straight-line distance an intruder can move within a sensor field without being detected. We will prove there is no strong barrier coverage in a $3 \mathrm{D}$ sensor network for a finite density of sensors when fixed emplacement sensors are uniformly dispersed during deployment. This necessitates the deployment of sensors with at least limited mobility. Finally, we develop an energy-efficient approach to use mobile sensors to construct a strong barrier in a 3D space.

This paper is organized as follows. In the next section, we summarize the related work. In Section III, we develop our network model. In Section IV, we introduce the notion of stealth distance for 3D sensor networks. In Section V, we demonstrate our results for strong barrier coverage on 3D sensor networks. In Section VI, we describe our approach to mitigating issues raised in the previous section. In Section VII, we describe our simulations and their results. Section VIII concludes the paper.

\section{RELATED WORK}

The problem of area coverage for wireless sensor networks has been studied intensively. Coverage of a grid-based sensor network was considered by [4]. The authors derived requirements on the sensing range and sensors failure rates to cover fully a given area. 
The problem of covering an arbitrary number of points with at least $k$ sensors is known as the $k$-Coverage Problem and has been well studied [5]-[7]. Research has been conducted to understand the relationship between area coverage and network connectivity [8], [9].

Power consumption is a crucial factor in the deployment of sensor networks. In many coverage scenarios, it may be possible to power down selected sensors while maintaining the required coverage. Scheduling sensor sleep cycles can extend network lifetime, which has been widely studied [10]-[16].

The notion of barrier coverage, whose goal is to arrange sensors to detect intruders passing through a region, was first described in the context of cooperating robots for military applications [17]. Examination of an intruder's ability to navigate through a sensor field undetected is a well studied problem [18]-[22]. Determining if within a belt region an intruder would be detected by at least $k$ sensors is known as the $k$-Barrier Coverage Problem [23], where the authors proposed algorithms to determine if a region is $k$-barrier covered, established deployment pattern to guarantee $k$-barrier coverage, and derived conditions of $k$-barrier coverage in a randomly deployed sensor network.

Covering a set of points to minimize the number of sensors employed to cover the area or maximize network lifetime is known as the Point Cover Problem and has been studied in [24]. The average distance an intruder can move through a $2 \mathrm{D}$ sensor field without being detected has been developed into a quality of surveillance metric [25].

Although not in a barrier context, the authors of [26] reference the use of magnetometer enabled motes to detect moving submarines at distances of several hundred meters. This seems reasonable given the sensitivity required to detect submarines using magnetometers and the current capabilities of magnetometer-based sensor boards for motes [27] [28].

\section{NeTwORK MOdEL}

We consider a sensor network consisting of sensors deployed in a large-scale 3-dimensional cuboid. For the initial configuration, we assume that the locations of these sensors are uniformly and independently distributed in the cuboid. Such a random initial deployment is desirable in scenarios where prior knowledge of the region of interest is not available, and may be the result of certain deployment strategies. Under this assumption, the sensor locations can be modeled by a stationary 3dimensional Poisson point process. Denote the density of the underlying Poisson point process as $\lambda$. The number of sensors located in a 3-dimensional region $R, N(R)$, follows a Poisson distribution with parameter $\lambda\|R\|$, where $\|R\|$ represents the volume of the region.

$$
\mathbb{P}(N(R)=k)=\frac{e^{-\lambda\|R\|}(\lambda\|R\|)^{k}}{k !} .
$$

We assume that each sensor can sense the environment and detect intruders in the 3-dimensional sphere of radius $r$. A point is said to be covered by a sensor if it is located in the sensing sphere of the sensor. The sensor network is thus partitioned into two parts, the covered space, which is the space covered by at least one sensor, and the uncovered space, which is the complement of the covered space. An intruder is said to be detected if its path it lies within the covered space.

Throughout the rest of this paper, the shorthand $X \sim$ $\exp (\mu)$ stands for $\mathbb{P}(X<x)=1-\exp (-\mu x)$, i.e., $X$ is a random variable exponentially distributed with parameter $\mu$.

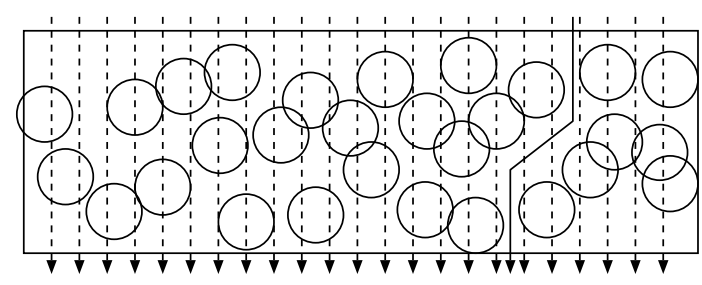

weak coverage

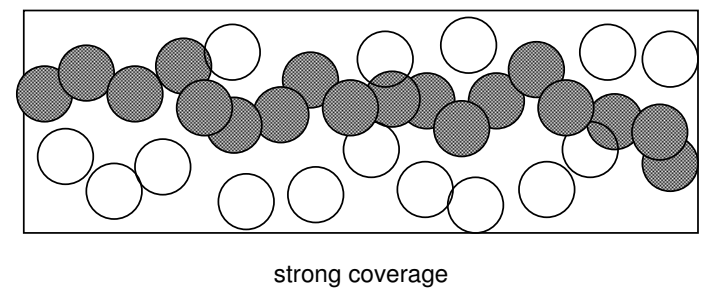

Fig. 1. Weak and strong barrier coverage.

In Figure 1 each circle represents covered space. Each path that extends from the top of the figure to the bottom of the figure through the sensor field represents a crossing path. Intruders moving along a straight line crossing path unaware of sensor positions will be detected even when the barrier does not provide complete coverage. With such weak barrier coverage, an intruder knowledgable of sensor positions can alter course to evade being detected. If we are able to construct a barrier so that no matter what path an intruder may take, he will be detected then our network is said to provide strong barrier coverage. It is easy to see that the gray circles in the second diagram form such a barrier. 


\section{Stealth Distance in 3D Sensor Networks}

In a wireless sensor network, as an intruder move through the network, it will be detected when intersecting with the sensing sphere of a sensor. We are interested in studying the distance an intruder travels before first being detected by sensors, as it measures the intrusion detection performance of the sensor network. This is illustrated in Figure 2. We formally define the stealth distance of an intruder as follows.

Definition 1: Stealth Distance. Consider a 3dimensional sensor network where sensors of sensing range $r$ are distributed according to a Poisson point process of density $\lambda$. Assuming that an intruder is initially undetected and moves in a random direction along a straight line. The stealth distance of the intruder, $X$, is defined as the distance it travels before first detected by any sensor.

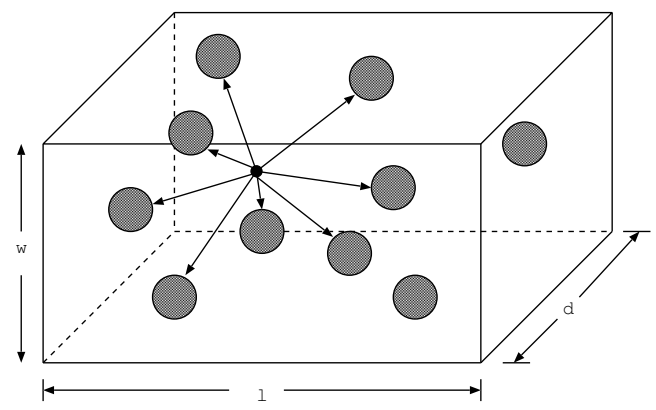

Fig. 2. Stealth distance of intruder in 3-dimensional sensor networks.

The notion of stealth distance has been introduced in [25], and the authors derived an approximation of the expected stealth distance for 2-dimensional sensor networks. In the following theorem, we characterize the distribution of the stealth distance in a 3-dimensional sensor network. The distribution of the stealth distance in a 2-dimensional sensor network can be obtained similarly.

Theorem 1: In a 3-dimensional sensor network where sensors are distributed according to a Poisson point process of density $\lambda$, the stealth distance of an intruder, $X$, follows an exponential distribution with parameter $\lambda \pi r^{2}$, i.e., $X \sim \exp \left(\lambda \pi r^{2}\right)$.

$$
P(X<x)=1-e^{-\lambda \pi r^{2} x}
$$

Proof: We first prove that for an intruder moving along a straight line in a random direction, the sequence of distances at which the intruder intersects a new sensor forms a Poisson process of intensity $\lambda \pi r^{2}$.

We denote by $V\left(l_{1}, l_{2}\right)$ the 3 -dimensional volume newly covered by the intruder when it moves from

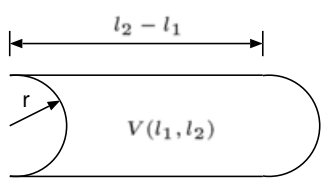

Fig. 3. Projection of volume $V\left(l_{1}, l_{2}\right)$ covered by an intruder when moving along a straight line.

location $l_{1}$ to $l_{2}$ along the straight line. The projection of the volume is illustrated in Figure 3.

First, we prove that the number of sensors that intersect the intruder during moving interval $\left[l_{1}, l_{2}\right]$ is Poisson distributed with parameter $\lambda \pi r^{2}\left(l_{2}-l_{1}\right)$. The probability that a sensor initially located at point $\mathbf{x} \in \mathbf{R}^{3}$ intersects the intruder within moving interval $\left[l_{1}, l_{2}\right]$ is $\mathbb{P}\left(\mathbf{x} \in \mathbf{V}\left(\mathbf{l}_{\mathbf{1}}, \mathbf{l}_{\mathbf{2}}\right)\right)$. This probability only depends on the direction and moving distance of the intruder; in particular, it does not depend on the initial Poisson process giving the positions of the sensors. We can thus define a thinned Poisson process $\Phi\left(l_{1}, l_{2}\right)$ by selecting the sensors that will intersect the intruder during the interval $\left[l_{1}, l_{2}\right]$. This process is non-uniform and has density

$$
\lambda^{\prime}(\mathbf{x})=\lambda \mathbb{P}\left(\mathbf{x} \in \mathbf{V}\left(\mathbf{l}_{\mathbf{1}}, \mathbf{l}_{\mathbf{2}}\right)\right) .
$$

The number of sensors intersecting the intruder within interval $\left[l_{1}, l_{2}\right]$ equals to the total number of points in the thinned process, which is Poisson distributed with mean

$$
\begin{aligned}
\mathbb{E}\left(\operatorname{card}\left(\Phi\left(l_{1}, l_{2}\right)\right)\right) & =\int_{\mathbb{R}^{3}} \lambda^{\prime}(\mathbf{x}) \mathbf{d} \mathbf{x} \\
& =\lambda \int_{\mathbb{R}^{3}} \mathbb{P}\left(\mathbf{x} \in \mathbf{V}\left(\mathbf{l}_{\mathbf{1}}, \mathbf{l}_{\mathbf{2}}\right)\right) \mathbf{d} \mathbf{x} \\
& =\lambda \int_{\mathbb{R}^{3}} \mathbb{E}\left(1_{\left\{\mathbf{x} \in \mathbf{V}\left(\mathbf{l}_{1}, \mathbf{l}_{2}\right)\right\}}\right) \mathbf{d x} \mathbf{x} \\
& =\lambda \mathbb{E}\left(\int_{\mathbb{R}^{3}} 1_{\left\{\mathbf{x} \in \mathbf{V}\left(\mathbf{l}_{1}, \mathbf{l}_{\mathbf{2}}\right)\right\}} \mathbf{d x}\right) \\
& =\lambda \mathbb{E}\left(\left\|V\left(l_{1}, l_{2}\right)\right\|\right),
\end{aligned}
$$

where $1_{\{\cdot\}}$ denotes the indicator function of the event $\{\cdot\}$. Furthermore, it is easy to see that $\mathbb{E}(\|V(l)\|)=\pi r^{2} l$.

Second, we show that the number of sensors hitting the intruder during disjoint moving intervals are independent. This is simply done by observing that if $\left[l_{1}, l_{2}\right] \cap\left[l_{3}, l_{4}\right]=$ $\emptyset$, each sensor is either selected in $\Phi\left(l_{1}, l_{2}\right)$ or in $\Phi\left(l_{3}, l_{4}\right)$ or not selected at all. Therefore, $\Phi\left(l_{1}, l_{2}\right)$ and $\Phi\left(l_{3}, l_{4}\right)$ are two independent processes.

Combining the two properties, we conclude that the sequence of distances at which the intruder intersects a new sensor is a Poisson process of intensity $\lambda \pi r^{2}$.

When the intruder intersects a sensor, it is immediately covered by the sensor until it moves out of the covering 
sphere of the sensor. There is no constraint on the number of sensors covering the intruder. Therefore, the covered/uncovered sequence experienced by the intruder can be seen as a $M / G / \infty$ queuing process, where the service time is the time duration that the intruder is covered by a sensor until it moves out of the sensors covering sphere. The idle periods of $M / G / \infty$ queue corresponds to the time duration that the point is not covered. It is known that idle periods in such queues have exponentially distributed durations. Therefore, we have

$$
X \sim \exp \left(\lambda \pi r^{2}\right)
$$

Based on Theorem 1, the expected stealth distance of a randomly located intruder is

$$
E[X]=\frac{1}{\lambda \pi r^{2}},
$$

which is inversely proportional to the sensor density $(\lambda)$ and the projected area of the sensing sphere $\left(\pi r^{2}\right)$. Therefore, in order to shorten the stealth distance of an intruder, one can add more sensors, or use sensors with larger sensing range. To guarantee that the expected stealth distance of an intruder be smaller than a specific threshold $l_{0}$, we should have

$$
\frac{1}{\lambda \pi r^{2}}<l_{0} \text {. }
$$

The above relationship between the stealth distance and the deployment parameters (sensor density and sensing range) provides important guidelines to the planning of sensor networks for intrusion detection.

\section{BArrier Coverage in 3D SEnsor Networks}

In a 2-dimensional sensor network, the barrier coverage of a strip area is directly related to the percolation of the network model, or more specifically, the existence and the number of disjoint connected sensor clusters that cross the strip. These connected sensor clusters act as "trip wires" that can detect any crossing intruders.

However, in a 3-dimensional sensor network, a sensor cluster connecting the opposite surfaces of the cuboid no longer constitutes a barrier that can detect crossing intruders. There could be "holes" in which an intruder can penetrate the cuboid undetected. Percolation of sensors no longer provides barrier coverage and intruders can evade from the uncovered space in the 3-dimensional space. This is illustrated in Figure 4, where the figure at the right-hand side shows the projection of the 3dimensional cuboid in the direction of the arrow.

We consider a 3-dimensional cuboid of size $l \times w \times d$, where $l, w$, and $d$ represents the length, width, and

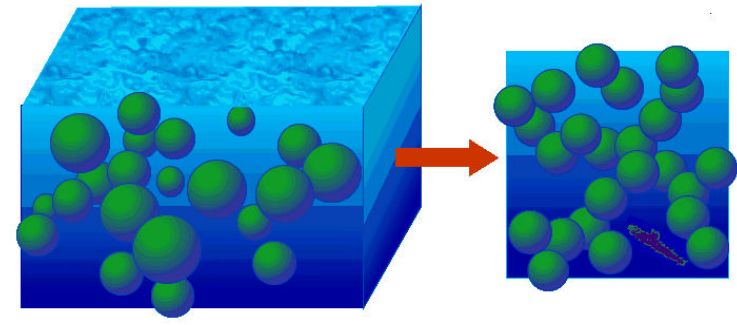

Fig. 4. Barrier coverage in 3-dimensional sensor networks
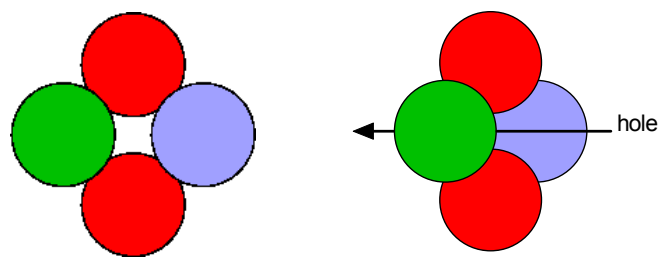

Fig. 5. Two different projections of the same sensors deployed in a 3D environment

depth of the cuboid, respectively. A crossing path is a path that connects one surface of the cuboid to the opposite surface, where the ingress point and the egress point reside on two opposite surfaces of the cuboid. A crossing path is said to be covered if it intercepts at least one sensor. Intruders moving along covered crossing paths will be detected. A network provides strong barrier coverage, if any crossing path attempted by an intruder results in his being detected.

A 3-dimensional sensor network is said to be strongly barrier covered in a specific direction if any crossing path intersecting the two surfaces perpendicular to the direction is covered.

Without loss of generality, we assume that intruders attempt to penetrate the cuboid in the direction of depth. Clearly, strong barrier coverage in the requires a continuous surface that is fully covered by sensors. The following theorem characterizes the strong barrier coverage in 3D space.

Theorem 2: In a 3-dimensional cuboid of size $l \times w \times d$ where sensors are distributed according to a Poisson point process of density $\lambda$, there is no strong barrier coverage in the direction of depth for finite sensor density $\lambda$ and depth $d$ as $l, w \rightarrow \infty$.

Proof. Projecting the 3-dimensional cuboid in the direction of the depth results in a 2-dimensional surface of size $l \times w$. After the projection, a "barrier tunnel" in the original 3-dimensional space where an intruder can pass through may not be detectable in the 2-dimensional projected surface. Figure 5 shows two different projections of four sensors deployed in a 3-dimensional environment. On the left-hand side we can see clearly a hole that can 
be used to form a barrier tunnel, but in the projection at the right-hand side, no hole is seen. Therefore, the barrier coverage of the 3-dimensional cuboid is bounded above by the barrier coverage of the projected 2-dimensional surface. If an intruder can penetrate the projected 2dimensional surface undetected, there exist uncovered crossing paths in the 3-dimensional cuboid.

In the asymptotic case, when $l, w \rightarrow \infty$, the sensors on the projected surface can be modeled as a 2-dimensional Poisson point process of density $\lambda d$. Based on [29][Theorem 1], the fraction of the area that is covered in the projected surface is

$$
1-e^{-\lambda d \pi r^{2}}<1 \text { if } \lambda, d<\infty .
$$

Therefore, there is uncovered area in the projected 2dimensional surface for finite sensor density and cuboid depth. As a result, there is no strong barrier coverage in the original 3-dimensional cuboid in the direction of depth.

\section{MOVING BARRIERS}

The results in the previous section show that strong barrier coverage cannot be accomplished with fixed emplacement sensors. We propose to employ mobile sensors to help construct a strong barrier. To accomplish this task, our proposed solution will require sensors with the following abilities: to move in all 3-dimensions underwater, communicate with other sensors, localize their own positions, and detect intruders.

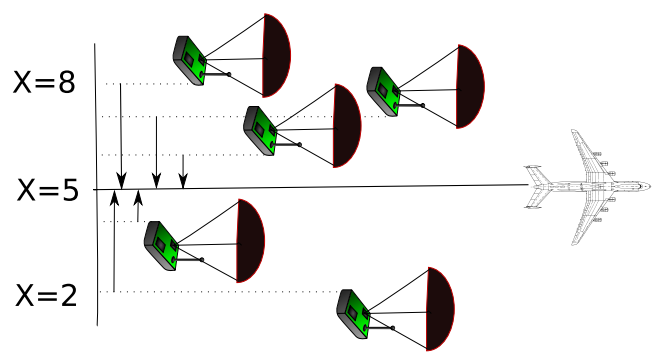

Fig. 6. Air drop of sensors along path resulting in scattered placement along that path.

We propose a three phased approach for constructing a strong 3-dimensional barrier, which minimizes the maximal energy expended by any one sensor:

- Find an optimal vertical plane at which the sensors will form a grid-based barrier.

- For each sensor, identify its optimal grid-based based assignment.

- Move sensors from their initial dropped position directly to their final assigned grid positions.

\section{A. Finding the optimal barrier location}

Suppose as in Figure 6 an airplane drops a set of mobile sensors such that the plane $X$ is parallel to the coast line to be protected. The sensors must move to some plane $X$, such that $X$ minimizes the energy expended by any one sensor. For this calculation, we are assuming that the sensors will only be moving in one direction to approach the $X$ location.

Suppose $X$ represents the optimal line for all sensors to approach. The straight-line distance traveled with any sensor $s_{i}$ in the direction of $X$. We are attempting to minimize the distance traveled $\min \sum_{i=1}^{n} d\left(x_{i}, x\right)$. To accomplish this let $x=\left(\max x_{i}-\min x_{i}\right) / 2$, then the maximum distance any one sensor travels is minimized. In Figure 6, all the sensors will move to the line $x=5$. It is straight forward to show that $X$ must be somewhere inside the set. As any two sensors move to meet one another, the total distance they travel is the distance between them. Thus, the sensors on either edge move the farthest, and half the distance between them minimizes the maximum distance any one sensor moves.

\section{B. Assignment of sensors to grid points}

Assigning $n$ sensors to $n$ grid positions is related to the Assignment Problem. In an instance of the Assignment Problem, we look to create a one-to-one matching between sensors and grid positions. There is a cost associated with each possible pairing of sensors to grid positions. In our application, the cost of each pairing is the energy required to move the sensor to its associated grid point. In the assignment problem, the goal is to minimize the total cost of the solution. A classic solution to this problem is known as the Hungarian Method and can be computed in $O\left(n^{3}\right)$, where $n$ is the number of sensors or grid positions [30]. The Hungarian Method takes two sets of objects, the costs associated with mapping all pairs, and it produces an one-to-one assignment which minimizes the total cost.

However, in our context, the goal it is not to minimize the total cost. We are looking to minimize the maximal amount of energy drain any one sensor incurs moving to a grid position. More formally, let $k$ be the maximal amount of energy we allow any sensor to expend for move from its current position to an arbitrary grid position. We seek to find the smallest value of $k$ such that each sensor expends no more energy than $k$, yet all sensors can move to cover a grid position.

We can apply the Hungarian Method to compute $k$. Assume we have a function $\operatorname{HungarianK}(S, G, k) \rightarrow$ Assignment, $k^{\prime}$. This function takes a list of sensor 
positions $S$, a list of grid positions $G$ which are to be covered, and a maximal value $k$. The function internally runs the traditional Hungarian algorithm. If all the edges in the intermediate result do not exceed $k$ then the assignment is returned. In addition $k^{\prime}$ represents the largest edge found during the assignment process. If some edge weight exceeds $k$ then the empty-set is returned.

With this new function a centralized solution can be accomplished at a central node as follows. Sensors transmit positional information to a central node. The central node creates a sorted list of edge weights. Since each sensor can be paired with every grid position the total number of edge weights constructed is $n^{2}$. A binary search is conducted on the lists indices. The values associated with the values of the midpoint indices are fed as the parameter $k$ to HungarianK.

During the binary search, an admissible solution will result in high being reduced and low is increased otherwise. Thus, the running time for a solution will be $O\left(n^{4}\right), O\left(n^{3}\right)$ for each running of Hungarian Method and $O\left(\lg n^{2}\right)$ or $O(n)$ for the binary search. Two practical improvements were made for this experiment. We used the index associated with the generated $k^{\prime}$ instead of the current high for the construction of the next midpoint as we know $k^{\prime}<=k$. During the sorting process, we remove duplicate edge weight values.

\section{Performance Evaluation}

We created a simulation to evaluate how the optimal solution would perform in a typical real world deployment. The average ocean depth is $3,790 \mathrm{~m}$. Specialized submarines like the Trieste have dived to $11,015 \mathrm{~m}$ [31]. More mainstream submarines like the Komsomolets series have dived to $1,300 \mathrm{~m}$ [32]. We decided that for our simulation we should propose a sensor network that would be able to detect submarines up to $3,790 \mathrm{~m}$.

Recent papers have stated that commercial motes with magnetometers can detect submarines at distances of several hundred meters [26]. For our simulation, we chose a fixed detection range of $320 \mathrm{~m}$. A column of six sensors with the first being deployed at a depth of $320 \mathrm{~m}$ allows coverage of $3840 \mathrm{~m}$. We allow for our sensors to sink from between $320 \mathrm{~m}$ to $3520 \mathrm{~m}$. The resulting cube's depth is $3200 \mathrm{~m}$. Modern submarines are capable of a submerged launch of anti-aircraft missiles [1]. This requires disperal from a high flying plane to minimize the risk of being shotdown during deployment. We allow sensors to drift away from the drop position with a radius up to $160 \mathrm{~m}$ in any direction, so a cube's width is $320 \mathrm{~m}$. Finally, each sensor column is $660 \mathrm{~m}$ apart. We assume the aircraft uniformly drops sensors from the location of the first column to the location of the final column. The length of a cube ranges from $1920 \mathrm{~m}$ to $55680 \mathrm{~m}$.

Our simulations compare the running time of the tradtional Hungarian Method and our variant HungarianK for different sized cubes. The former computes the minimum total weight solution and the latter computes the minimum maximum edge weight. All of our simulations were run on an Intel T2250 1.73GHz CPU. The operating system was Red Hat Fedora Core 8. All software was written in Python. Figure 7 depicts our results. The

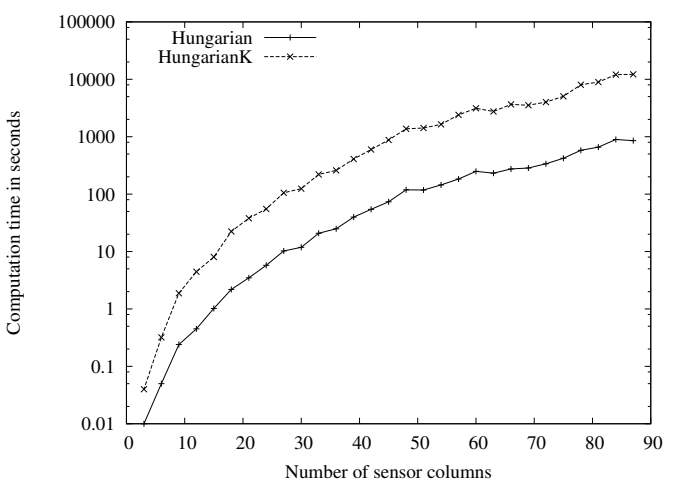

Fig. 7. Comparison of running time between the Hungarian and with our HungarianK variant.

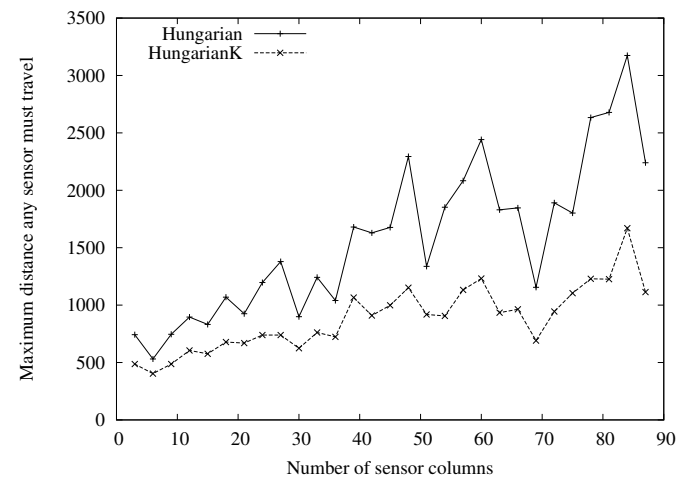

Fig. 8. Comparing the Hungarian Method with our HungarianK variant to determine maximum moving distance.

$\mathrm{X}$-axis represents the number of sensor columns being tested, which each sensor column being comprised of six sensors. The y-axis represents the total computation time required to achieve a result. This graphic shows that the computation time for computing HungarianK is within $O\left(n^{4}\right)$. However, this is not a scalable solution.

Figure 8 depicts the maximum movement any one sensor must travel to get to its optimal assignment. The $\mathrm{x}$-axis is as before. The $\mathrm{y}$-axis represents the maximum distance any one sensor must travel. As the network size increases the Hungarian Method requires move movement from sensors. Figures 7,8 show there is a tradeoff 
to be made between computation time/energy and total movement distance. This is an important consideration in energy constrained environments where movement is far more pricey then computation.

\section{CONCLUSIONS AND FUture WORK}

In this work, we used an optimal centralized solution to construct our barrier. The solution resulted in the minimum energy being expended for construction. The solution proposed is not scalable to large sensor deployments. We plan to investigate distributed approximate solutions which will hopefully produce similarly efficient results which will be scalable to larger sensor grids.

\section{ACKNOWLEDGEMENT}

Benyuan Liu was supported in part by NSF under grant CNS-0721626. Jie Wang was supported in part by NSF under grants CNS-0709001 and CCF-0830314.

\section{REFERENCES}

[1] J. Kong, J. Kong, J.-h. Cui, D. Wu, and M. Gerla, "Building underwater ad-hoc networks and sensor networks for large scale real-time aquatic applications," in Proc. IEEE Military Comm. Conf. MILCOM 2005, J.-h. Cui, Ed., 2005, pp. 1535-1541 Vol. 3.

[2] (2008) Global submarine proliferation: Emerging trends and problems. [Online]. Available: http://www.nti.org/e_research/ e3_74.html

[3] H. Gharavi, H. Gharavi, and S. Kumar, "Special issue on sensor networks and applications," Proc. of the IEEE, vol. 91, no. 8, pp. 1151-1153, 2003.

[4] S. Shakkottai, R. Srikant, and N. Shroff, "Unreliable sensor grids: Coverage, connectivity and diameter," in Proc. IEEE Infocom, 2003.

[5] C. Huang and Y. Tseng, "The coverage problem in a wireless sensor network," in ACM Internat. Workshop on Wireless Sensor Networks and Appl. (WSNA), 2003, pp. 115-121.

[6] Z. Zhou, S. Das, and H. Gupta, "Connected k-coverage problem in sensor networks," in Intl. Conf. on Computer Comm. and Networks (ICCCN), 2004.

[7] S. Kumar, T. Lai, and J. Balogh, "On k-coverage in a mostly sleeping sensor network," in Proc. of ACM Mobicom, 2004.

[8] X. Wang, G. Xing, Y. Zhang, C. Lu, R. Pless, and C. Gill, "Integrated coverage and connectivity configuration in wireless sensor networks," in ACM Conf. on Embedded Networked Sensor Systems (SenSys'03), 2003.

[9] H. Zhang and J. Hou, "Maintaining sensing coverage and connectivity in sensor networks," in invited paper in Internat. Workshop on Theoret. and Algorithmic Aspects of Sensor, Ad Hoc Wireless and Peer-to-Peer Networks, 2004.

[10] D. Tian and N. D. Georganas, "A coverage-preserving node scheduling scheme for large wireless sensor networks," in First ACM Internat. Workshop on Wireless Sensor Networks and Appl. (WSNA), 2002, pp. 32-41.

[11] F. Ye, G. Zhong, S. Lu, and L. Zhang, "Peas: A robust energy conserving protocol for long-lived sensor networks," in Proc. ICDCS, 2003.
[12] T. Yan, T. He, and J. A. Stankovic, "Differentiated surveillance for sensor networks," in Proc. of ACM Sensys, 2003.

[13] C. fan Hsin and M. Liu, "Network coverage using low dutycycled sensors: Random and coordinated sleep algorithms," in Proc. Internat. Workshop on Inform. Processing in Sensor Networks (IPSN), 2004.

[14] Z. Abrams, A. Goel, and S. Plotkin, "Set k-cover algorithms for energy efficient monitoring in wireless sensor networks," in Proc. Internat. Workshop on Inform. Processing in Sensor Networks (IPSN), 2004.

[15] J. Wu and S. Yang, "Coverage issue in sensor networks with adjustable ranges," in Internat. Workshop on Mobile and Wireless Networking (MWN), 2004.

[16] G. Lu, N. Sadagopan, B. Krishnamachari, and A. ish Goel, "Delay efficient sleep scheduling in wireless sensor networks," in Proc. IEEE Infocom, 2005.

[17] D. Gage, "Command control for many-robot systems." in Proc. of the Nineteenth Annual AUVS Tech. Sympos. (AUVS-92), 1992.

[18] S. Meguerdichian, F. Koushanfar, M. Potkonjak, and M. B. Srivastava, "Coverage problems in wireless ad-hoc sensor networks," in Proc. IEEE Infocom, 2001, pp. 1380-1387.

[19] S. Meguerdichian, F. Koushanfar, G. Qu, and M. Potkonjak, "Exposure in wireless ad-hoc sensor networks," in ACM Mobile Computing and Networking, 2001, pp. 139-150.

[20] P.-J. W. X.-Y. Li and O. Frieder, "Coverage in wireless ad-hoc sensor networks," IEEE Transactions on Computers, vol. 52(6), pp. 753-763, June 2003.

[21] G. Veltri, Q. Huang, G. Qu, and M. Potkonjak, "Minimal and maximal exposure path algorithms for wireless embedded sensor networks," in Proc. of ACM Sensys, 2003.

[22] B. Liu, O. Dousse, J. Wang, and A. Saipulla, "Strong barrier coverage of wireless sensor networks," in Proc. of Mobihoc '08.

[23] T. H. L. S. Kumar and A. Arora, "Barrier coverage with wireless sensors," in Proc. ACM Mobicom, 2005.

[24] M. Cardei and J. Wu., Handbook of Sensor Networks. CRC Press, 2004, ch. Coverage in Wireless Sensor Networks.

[25] C. Gui and P. Mohapatra, "Power conservation and quality of surveillance in target tracking sensor networks," 2004.

[26] T. Nguyen, T. Nguyen, D. Nguyen, and D. Tran, "Stochastic binary sensor networks for noisy environments," in Proc. First Internat. Conf. on Comm. and Electronics ICCE '06, D. Nguyen, Ed., 2006, pp. 41-46.

[27] J. Lenz, H. Inc, and M. Minneapolis, "A review of magnetic sensors," pp. 973-989, 1990.

[28] (2008) 1- and 2-axis magnetic sensors. [Online] Available: http://www.ssec.honeywell.com/magnetic/datasheets/ hmc1001-2_1021-2.pdf

[29] B. Liu and D. Towsley, "A study on the coverage of large-scale sensor networks," in The 1st IEEE Internat. Conf. on Mobile Ad-hoc and Sensor Systems, 2004.

[30] H. Kühn, "The Hungarian Method for the Assignment Problem," Naval Research Logistics Quarterly, vol. 2, pp. 83-97, 1955.

[31] (2006) Seven miles down: The story of the bathyscaph trieste. [Online]. Available: http://bjsonline.com/watches/articles/0022_ 3.shtml

[32] (2008) Russia685. [Online]. Available: http://www. globalsecurity.org/military/world/russia/685-specs.htm 